\title{
School Daze: Capturing Chemical Exposures in Syndromic Surveillance - New Jersey 2013
}

\author{
Teresa Hamby*1, Victor Pomary ${ }^{1}$, Eric Adler², Stella Tsai ${ }^{1}$ and Andrew Walsh ${ }^{3}$ \\ ${ }^{1}$ New Jersey Department of Health, Trenton, NJ, USA; ${ }^{2}$ Atlantic County (NJ) Division of Public Health, Atlantic City, NJ, USA; ${ }^{3} \mathrm{Health}$ \\ Monitoring Systems, Inc, Pittsburgh, PA, USA
}

\section{Objective}

To describe the development of a new chemical exposure classifier in New Jersey's syndromic surveillance system (EpiCenter).

\section{Introduction}

In early May of 2013, two chemical spills occurred within high schools in Atlantic county. These incidents, occurring within a week of each other, highlighted the need to strengthen statewide syndromic surveillance of illnesses caused by such exposures. In response to these spills, a new 'chemical exposure' classifier was created in EpiCenter, New Jersey's syndromic surveillance system, to track future events by monitoring registration chief complaint data taken from emergency department visits. The primary objective behind creation of the new classifier is to provide local epidemiologists with prompt notification once EpiCenter detects an abnormal numbers of chemical exposure cases.

\section{Methods}

In New Jersey, 75 of 81 total acute care and satellite Emergency Departments (EDs) are connected to EpiCenter, an online syndromic surveillance system developed by Health Monitoring Systems, Inc (HMS) that incorporates statistical management and analytical techniques to process health-related data in real time. Chief complaint text is classified, using text recognition methods, into various public health-related and other categories. EpiCenter chief complaint data for the period of March 1, 2013 through June 10, 2013 was exported and analyzed to assist in the development of the search algorithm and to determine the background level of chemical exposures typically reported by New Jersey emergency departments.

\section{Results}

Analysis of the data incorporating the new classifier for the period of March 1, 2013 through June 10, 2013 revealed that on average, most counties reported 0-1 cases of chemical exposure per day. Additionally, three densely-populated $\mathrm{NJ}$ counties, including the county where the exposures occurred, reported the highest total number of chemical exposure cases $(40,39$ and 30$)$. Two counties, one north and one south, were the only counties that did not report any cases over the observed time period.

\section{Conclusions}

The newly-created classifier is currently configured to send an immediate notification to local epidemiologists once two cases are recorded within a 24-hour window ending at midnight each day. Given that background levels across the state are around 0-1 cases per day, an alert threshold of 2 cases is expected to be sensitive enough to flag potential chemical exposure threats for further investigation without burdening local epidemiologists with excessive notifications. These chemical exposure events, which resulted in the development of a new classifier, highlight the importance of setting investigation- generating alert thresholds within EpiCenter at a level that will minimize "false" positives without risking the missing of "true" events requiring additional attention.

\section{Keywords}

Chemical Exposure; Syndromic Surveillance; Classification; EpiCenter; New Jersey

\section{*Teresa Hamby}

E-mail: Teresa.Hamby@doh.state.nj.us 